\title{
Physical and Mechanical Properties of Resins Blends Containing a Monomethacrylate with Low-polymerization Shrinkage
}

\author{
Aurealice Rosa Maria Martins ${ }^{1} \quad$ Luciana Machado-Santos ${ }^{2}$ Regis Cleo Fernandes Grassia Jr ${ }^{3}$ \\ Rafael Pino Vitti ${ }^{2,4}$ Mário Alexandre Coelho Sinhoreti ${ }^{1}$ William Cunha Brandt ${ }^{3}$
}

1Department of Restorative Dentistry, Piracicaba Dental School, University of Campinas, Piracicaba, SP, Brazil

2Department of Prosthodontics, School of Dentistry, University of Taubaté, Taubaté, SP, Brazil

${ }^{3}$ School of Dentistry, Santo Amaro University, São Paulo, SP, Brazil

${ }^{4}$ School of Dentistry, Herminio Ometto University Center, Araras, SP, Brazil

Eur J Dent:2021;5:96-100

\begin{abstract}
Address for correspondence William Cunha Brandt, DDS, MSc, PhD, Santo Amaro University - School of Dentistry, Implantology Area, Rua Professor Eneas de Siqueira Neto, 340, São Paulo-SP 04829-300, Brazil (e-mail: williamcbrandt@yahoo.com.br).
\end{abstract}

\begin{abstract}
Keywords

- methacrylate monomers

- dental resin

- curing performance
\end{abstract}

Objectives The aim of this study was to evaluate the Knoop hardness (KH), cross-link density (CLD), water sorption (WS), water solubility (WSB), and volumetric shrinkage (VS) of experimental resins blends containing a monomethacrylate with low-polymerization shrinkage.

Materials and Methods A blend of bisphenol glycidyl methacrylate (BisGMA) as base monomer was formulated with (Bis-GMA)/triethyleneglycol dimethacrylate (TEGDMA), Bis-GMA/isobornyl methacrylate (IBOMA), or Bis-GMA/TEGDMA/IBOMA in different concentrations $(40,50$, or 60 wt\%). The camphorquinone (CQ)/2-(dimethylamino) ethyl methacrylate (DMAEMA) was used as the photoinitiator system. The KH and CLD were measured at the top surface using an indenter. For WS and WSB, the volume of the samples was calculated in $\mathrm{mm}^{3}$. The samples were transferred to desiccators until a constant mass was obtained $(\mathrm{m} 1$ ) and were subsequently immersed in distilled water until no alteration in mass was detected $(\mathrm{m} 2)$. The samples were reconditioned to constant mass in desiccators (m3). WS and WSB were determined using the equations $m 2-m 3 / V$ and $m 1-m 3 / V$, respectively. VS results were calculated with the density parameters before and after curing.

Statistical Analysis Data were submitted to ANOVA and Tukey's test $(\alpha=0.05)$.

Results The resins containing IBOMA showed lower VS results. TEGDMA $40 \%$ and TEGDMA/IBOMA 20/20 wt\% showed higher KH values. The IBOMA groups showed lower CLD, while TEGDMA groups had higher values of CLD. The BisGMA/TEGDMA resin presented the highest values of WS, and for WSB, all groups showed no significant differences among themselves.

Conclusion The monomethacrylate with low-polymerization shrinkage IBOMA used alone or in combination with TEGDMA may decrease VS, WS, and CLD values.
DoI https://doi.org/ 10.1055/s-0040-1716985 ISSN 1305-7456.
(C) 2021. European Journal of Dentistry.

This is an open access article published by Thieme under the terms of the Creative Commons Attribution-NonDerivative-NonCommercial-License, permitting copying and reproduction so long as the original work is given appropriate credit. Contents may not be used for commercial purposes, or adapted, remixed, transformed or built upon. (https://creativecommons.org/licenses/by-nc-nd/4.0/) Thieme Medical and Scientific Publishers Pvt. Ltd., A-12, 2nd Floor, Sector 2, Noida-201301 UP, India 


\section{Introduction}

Composite resins are highly successful restorative materials in dentistry. ${ }^{1,2}$ However, there are factors concerning the deleterious processes caused by the stress generated during the polymerization reaction. ${ }^{1}$ Dental structures are routinely restored with dental restorative materials for aesthetic or functional problems caused by several factors such as tooth decay and traumas. The restorative materials represent one of the many successes of modern dental research in biomaterials. ${ }^{1-3}$

Dental composites are derived from methacrylate networks, and the base monomer most currently used in these composites is bisphenol glycidyl methacrylate (Bis-GMA), known to be somewhat volatile, low diffusivity between tissues and low shrinkage. ${ }^{2}$ The high viscosity of Bis-GMA monomers requires the addition of low-molecular weight monomer to obtain a suitable viscosity and improve mobility of the monomers during the polymerization reaction, increasing the degree of conversion ${ }^{4,5}$, in addition to providing the incorporation of inorganic particle fillers. 4,6

Due to its low viscosity and ability to increase the degree of conversion, triethyleneglycol dimethacrylate (TEGDMA) is a diluent monomer widely added to the base monomer. ${ }^{4,7}$ However, TEGDMA has high volumetric shrinkage (VS). ${ }^{8}$ Still, the addition of diluent TEGDMA in greater proportion increases the polymerization shrinkage and water WS of the matrix, ${ }^{9}$ potentially leading to gap formation, marginal pigmentation, and secondary caries. Many alternative monomers with reactive diluents' intention of partial or total substitution of TEGDMA have often been exploited as a mean to reduce these problems. ${ }^{10,11}$

Studies have been developed to evaluate physical and mechanical properties of resins blends. ${ }^{10-16}$ The isobornyl methacrylate (IBOMA) is a monomethacrylate that has low viscosity and polymerization shrinkage. Studies show that their use in synthesizing nanogels for matrix resins aiming to reduce shrinkage and polymerization stress ${ }^{15,16}$ is also used as comonomers thinners matrix composites because of their low viscosity, low polymerization shrinkage, and high hydrophobicity. ${ }^{17}$ Besides, this comonomer has low water WS, which can increase the durability of the polymer due to the resistance to degradation, particularly in the oral environment. ${ }^{4,7}$

The phenomena of WS and WSB may be precursors to a variety of chemical and physical processes that promote biological concerns and have deleterious effects on the structure and function of resin matrixes. ${ }^{18}$ Polymer structure quality such as the degree of conversion and cross-link density (CLD) resulting from the photoactivation mode may lead to differences in WS and WSB. ${ }^{4,7,19}$

Thus, it would be interesting to know the potential of experimental resin blends for dental resins. The aim of this study was to evaluate the physical and mechanical properties of experimental resin blends using Bis-GMA as monomer base, IBOMA as reactive diluent monomer alone or in combination with TEGDMA with different proportions. The hypothesis tested in this study is that the addition of alternative diluent monomer (IBOMA) may decrease the water WS and WSB and improve the $\mathrm{KH}$ and $\mathrm{CLD}$ of experimental resins.

\section{Materials and Methods}

\section{Resin Preparation}

Nine experimental resin formulations were tested in this study. The resin matrix consisted of bisphenol glycidyl methacrylate (BisGMA - Sigma-Aldrich Inc, St Louis, MO, USA) as base monomer and two diluent reactive co-monomers: triethyleneglycol dimethacrylate (TEGDMA - Sigma-Aldrich Inc, St Louis, MO, USA) and isobornyl methacrylate (IBOMA - Sigma-Aldrich Inc, St Louis, MO, USA). The structure of these molecules of monomers is showed in the - Fig. $\mathbf{1}$. The diluent comonomers were mixed with the base monomers in nine different proportions, as described in - Table $\mathbf{1}$. The photoinitiator system was composed of camphorquinone (CQ-0.5 wt\%, Sigma-Aldrich Inc, St Louis, MO, USA and 2-(dimethylamino) ethyl methacrylate (DMAEMA-1 wt\%; Sigma-Aldrich Inc, St Louis, MO, USA). Also, the inhibitor BHT (butylated hydroxytoluene; Sigma-Aldrich Inc, St Louis, MO, USA) was added to the organic matrix in a concentration of $0.1 \mathrm{wt} \%$ to avoid spontaneous polymerization of the monomers. ${ }^{11}$<smiles>C=C(C)C(=O)OCC(O)COc1ccc(C(C)(C)c2ccc(OCC(O)COC(=O)C(=C)C)cc2)cc1</smiles><smiles>C=C(C)C(=O)OCCOCCOCCOC(=O)C(=C)C</smiles>

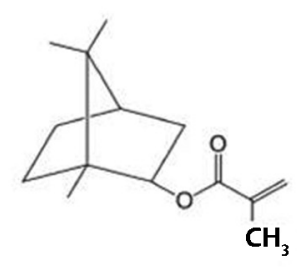

IBOMA

Fig. 1 Bisphenol glycidyl methacrylate (Bis-GMA), triethyleneglycol dimethacrylate (TEGDMA), and isobornyl methacrylate (IBOMA) molecule.

Table 1 Composition of the experimental resin blends

\begin{tabular}{|l|l|l|l|}
\hline Resin & Bis-GMA\% & TEGDMA\% & IBOMA\% \\
\hline Bis50-TEG50 & 50 & 50 & - \\
\hline Bis60-TEG40 & 60 & 40 & - \\
\hline Bis40-TEG60 & 40 & 60 & - \\
\hline Bis50-IBO50 & 50 & - & 50 \\
\hline Bis60-IBO40 & 60 & - & 40 \\
\hline Bis40-IBO60 & 40 & - & 60 \\
\hline Bis50-TEG25-IBO25 & 50 & 25 & 25 \\
\hline Bis60-TEG20-IBO20 & 60 & 20 & 20 \\
\hline Bis40-TEG30-IBO30 & 40 & 30 & 30 \\
\hline
\end{tabular}

Abbreviations: Bis-GMA, bisphenol glycidyl methacrylate; IBOMA, isobornyl methacrylate; TEGDMA, triethyleneglycol dimethacrylate. 


\section{Knoop Hardness (KH)}

For the KH test, circular samples $(n=10)$ were prepared ( $2 \mathrm{~mm}$ thickness $\times 5 \mathrm{~mm}$ diameter) and light cured by a LED curing unit (Bluephase $\mathrm{G} 2,1200 \mathrm{~mW} / \mathrm{cm}^{2}$ ) over $60 \mathrm{~s}$. The total energy dose was standardized at $72 \mathrm{~J}$. After light-curing procedures, the specimens were dry stored at $37^{\circ} \mathrm{C}$ for 24 hours in light-proof containers. Thereafter, the top surface was wet-polished with 1,200-grit SiC paper to obtain a planar surface. KH measurements were taken using an indenter (HMV-2, Shimadzu, Tokyo, Japan), under a load of $490 \mathrm{~N}$ for 15 seconds. Five readings were performed for each specimen. The Knoop hardness number (KHN, in kilogram-force per square millimeter) was recorded as the average of the five indentations. Data were submitted to one-way ANOVA test followed by Tukey's test $(\alpha=0.05)$.

\section{Cross-link Density (CLD)}

After completion of the KH test, samples of each resin $(n=10)$ were used to test CLD. These samples were immersed in $100 \%$ ethanol, for 24 hours, to indirectly evaluate the CLD and the elution of monomers by $\mathrm{KH}$ mean. The $\mathrm{KH}$ measurements were taken on top surface using an indenter (HMV-2, Shimadzu, Tokyo, Japan) under a load of $490 \mathrm{~N}$ (equivalent to 50 gf) for 15 seconds. Five readings were performed for each sample. The KHN was recorded as the average of the five indentations. Data were submitted to the one-way ANOVA test, followed by Tukey's test $(\alpha=0.05$ ). Additionally, the percentage decrease values of $\mathrm{KH}$ obtained for each experimental resin was calculated.

\section{Sorption and Solubility}

This study was performed in compliance with ISO 4049: $2000^{20}$ standard specifications (except for the specimen dimensions and curing protocol) as follows: To test the WS and WSB, circular samples $(2 \mathrm{~mm}$ thickness $\times 5 \mathrm{~mm}$ diameter) were prepared ( $n=5)$ and light cured by LED curing unit (Bluephase G2, $1200 \mathrm{~mW} / \mathrm{cm}^{2}$ ) over $60 \mathrm{~s}$. The total energy dose was standardized at $72 \mathrm{~J}$. The disks were stored in desiccators containing silica gel at $37^{\circ} \mathrm{C}$. The samples were weighted daily in an analytical balance (Tel Marke, Bel Quimis, São Paulo, SP, Brazil), accurate to $0.001 \mathrm{mg}$, constituting a weighing cycle every 24 hours. The complete cycle was repeated until a constant mass (m1) was obtained (2 days of no weight change). Thickness (four measurements at four equidistant points on the circumference) and diameter (two measurements at the right angles) of each specimen were measured using a digital electronic caliper (Mitutoyo Corporation, Tokyo, Japan). The mean values were used to calculate the volume (V) of each specimen (in $\mathrm{mm}^{3}$ ). Thereafter, the samples were stored in plastic containers with distilled water at $37^{\circ} \mathrm{C}$ for 7 days. The volume of immersion water was $6 \mathrm{~mL}$ per specimen. Samples were again weighted daily after being carefully wiped with an absorbent paper. When constant weight was obtained (2 days of no weight change), this value was recorded as $\mathrm{m} 2$. After this weighing, the samples were returned to the first desiccator. The entire mass reconditioning cycle was repeated and the constant mass (2 days of no weight change) was recorded as $\mathrm{m} 3$. The values for WS and WSB, in micrograms per cubic millimeters, were calculated using the following equations:

$$
\begin{aligned}
W S & =(m 2-m 3) / V \\
W S B & =(m 1-m 3) / V
\end{aligned}
$$

\section{Volumetric Shrinkage (VS)}

The VS was determined by measuring the resin density before $\left(\rho_{\mathrm{u}}\right)$ and after $\left(\rho_{\mathrm{c}}\right)$ light curing $(n=10)$ with the help of Archimedes' principle. The mass $(\mathrm{m})$ of the uncured sample was measured on a precision balance, the volume $(v)$ was measured with a pipette, and the initial density $\left(\rho_{\mathrm{u}}\right)$ was calculated as follows:

$$
\rho_{\mathrm{u}}=\mathrm{m} / \mathrm{v}
$$

After light curing was performed for $60 \mathrm{~s}$ (Bluephase G2, $1200 \mathrm{~mW} / \mathrm{cm}^{2}$ ), the final mass of the sample was measured in air and water, and the final density $\left(\rho_{c}\right)$ was calculated. The VS measurement was made after 24 hours of dry storage at $37^{\circ} \mathrm{C}$. The VS (vol\%) was calculated by the following equation:

$$
\mathrm{VS}=\left(\rho_{\mathrm{c}}-\rho_{\mathrm{u}} / \rho_{\mathrm{c}}\right) \times 100
$$

where $\rho_{c}$ is the final density (cured) and $\rho_{u}$ is the initial density (uncured). ${ }^{15}$

\section{Statistical Analyses}

The data were analyzed by one-way ANOVA and posthoc Tukey's tests. Statistical significance was established at $\alpha=0.05$ for all tests.

\section{Results}

The KH and CLD values are shown in - Table 2 and -Fig. 2 . The resin Bis-GMA/TEGDMA 60/40\% by weight and Bis-GMA/ TEGDMA/IBOMA $60 / 20 / 20 \%$ by weight showed the highest values of $\mathrm{KH}$, and Bis-GMA/IBOMA 40/60 wt\% showed the lowest values. The IBOMA groups had the lowest means for CLD, while the TEGDMA groups showed higher values of CLD. The groups of resins where the two reactive diluents monomers were present had intermediate values. The hardness decrease for TEGDMA groups ranged from 45.65 to 54.92\%; for IBOMA groups, it ranged from 74.05 to $81.02 \%$; for TEGDMA-IBOMA groups, it ranged from 63.66 to $69.39 \%$.

The WS and WSB data are listed in - Table 3. The resins that had TEGDMA as diluent monomer showed the highest values of WS, and for WSB, all groups showed no significant differences among themselves.

The VS data are listed in - Table 4 . The resins that had IBOMA as diluent monomer showed the lowest values of VS.

\section{Discussion}

The hypothesis was rejected, because IBOMA used as monomer diluent showed lower VS and WS values, but similar WBS values and lower $\mathrm{KH}$ and CLD values when compared with 
Table 2 Means and standard deviation of the KHN, CLD (KHN), and hardness decrease (\%) for the experimental resin blends

\begin{tabular}{|l|l|l|l|}
\hline Resins & Hardness (KHN) & CLD (KHN) & $\%$ \\
\hline Bis50-TEG50 & $31.1(4.1) \mathrm{AB}$ & $16.9(1.8) \mathrm{A}$ & 45.65 \\
\hline Bis60-TEG40 & $35.5(4.8) \mathrm{A}$ & $16.0(2.0) \mathrm{A}$ & 54.92 \\
\hline Bis40-TEG60 & $31.4(5.7) \mathrm{AB}$ & $16.8(2.0) \mathrm{A}$ & 46.49 \\
\hline Bis50-IBO50 & $26.7(3.7) \mathrm{BC}$ & $6.6(0.9) \mathrm{C}$ & 75.28 \\
\hline Bis60-IBO40 & $31.1(3.8) \mathrm{AB}$ & $5.9(0.6) \mathrm{C}$ & 81.02 \\
\hline Bis40-IBO60 & $23.9(2.8) \mathrm{C}$ & $6.2(0.6) \mathrm{C}$ & 74.05 \\
\hline Bis50-TEG25-IBO25 & $31.1(3.3) \mathrm{AB}$ & $10.4(0.9) \mathrm{B}$ & 66.55 \\
\hline Bis60-TEG20-IBO20 & $33.0(2.2) \mathrm{A}$ & $10.1(0.6) \mathrm{B}$ & 69.39 \\
\hline Bis40-TEG30-IBO30 & $32.2(4.5) \mathrm{AB}$ & $11.7(0.9) \mathrm{B}$ & 63.66 \\
\hline
\end{tabular}

Abbreviations: cross-link density (CLD); KHN, Knoop hardness.

Distinct letters are statistically different for each column $(\rho<0.05)$.

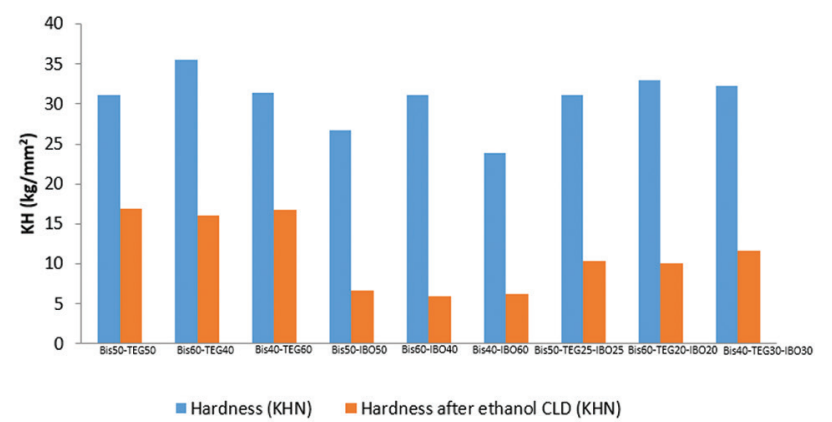

Fig. 2 Graphic presenting the cross-link density survey with the Knoop hardness before and after immersion in absolute ethanol. Knoop hardness reduction (\%) presented above the columns.

Table 3 Means and standard deviation of the water sorption and solubility for the experimental resin blends

\begin{tabular}{|l|l|l|}
\hline Resins & WS & WSB \\
\hline Bis50-TEG50 & $41.4(8.5) \mathrm{A}$ & $1.28(2.86) \mathrm{A}$ \\
\hline Bis60-TEG40 & $35.5(5.1) \mathrm{A}$ & $3.23(6.76) \mathrm{A}$ \\
\hline Bis40-TEG60 & $48.4(4.2) \mathrm{A}$ & $2.46(6.88) \mathrm{A}$ \\
\hline Bis50-IBO50 & $15.3(3.2) \mathrm{C}$ & $5.76(8.35) \mathrm{A}$ \\
\hline Bis60-IBO40 & $22.9(6.2) \mathrm{BC}$ & $3.52(3.22) \mathrm{A}$ \\
\hline Bis40-IBO60 & $15.4(4.7) \mathrm{C}$ & $10.17(8.08) \mathrm{A}$ \\
\hline Bis50-TEG25-IBO25 & $29.2(4.2) \mathrm{B}$ & $6.47(8.85) \mathrm{A}$ \\
\hline Bis60-TEG20-IBO20 & $26.2(5.3) \mathrm{BC}$ & $2.59(7.13) \mathrm{A}$ \\
\hline Bis40-TEG30-IBO30 & $24.6(4.8) \mathrm{BC}$ & $4.63(5.37) \mathrm{A}$ \\
\hline & & $\rho=0.1559$ \\
\hline
\end{tabular}

Abbreviations: WSB, water solubility; WS, water sorption.

Bis-GMA/TEGDMA resins. According to the results obtained, it can be verified that the IBOMA monomer decreased the VS values of the experimental dental resins. Overall, the resins with IBOMA (R5, R6, R7 and R9) showed lower VS values when compared with traditional BisGMA/TEGDMA dental resins, showing the potential for decreasing the polymerization shrinkage of IBOMA.
Table 4 Means and standard deviation for the VS\% for the experimental resin blends

\begin{tabular}{|l|l|}
\hline Resins & VS (\%) \\
\hline Bis50-TEG50 & $7.17(0.36) \mathrm{BC}$ \\
\hline Bis60-TEG40 & $7.51(0.23) \mathrm{AB}$ \\
\hline Bis40-TEG60 & $8.36(0.27) \mathrm{A}$ \\
\hline Bis50-IBO50 & $6.36(0.37) \mathrm{CD}$ \\
\hline Bis60-IBO40 & $4.03(0.47) \mathrm{F}$ \\
\hline Bis40-IBO60 & $3.90(0.97) \mathrm{F}$ \\
\hline Bis50-TEG25-IBO25 & $5.06(0.26) \mathrm{E}$ \\
\hline Bis60-TEG20-IBO20 & $6.37(0.57) \mathrm{CD}$ \\
\hline Bis40-TEG30-IBO30 & $5.72(0.24) \mathrm{DE}$ \\
\hline
\end{tabular}

Abbreviation: VS, volumetric shrinkage.

Distinct letters are statistically different $(\rho<0.05)$.

WSB in resin-based materials is a diffusion-controlled process and occurs mainly in the resin matrix. ${ }^{21}$ In this study, it was high values of WS were observed when the reactive diluent monomer TEGDMA was present in the resin matrix. Higher TEGDMA content in the matrix is responsible for increasing the WS of the composites. ${ }^{22}$ The WS of the copolymer is influenced by the hydrophilicity ${ }^{23,24}$ and CLD of the copolymer. ${ }^{25}$

The influence of the composition on CLD of experimental composites containing different variations of TEGDMA/ Bis-GMA, using hardness test before and after immersion in absolute ethanol, was examined. They observed that the variation in the composite composition influenced the CLD. ${ }^{26}$ Also, a decrease in the hydrophilicity and an increase in the CLD of a copolymer could reduce the WS of the matrix. ${ }^{23,24}$ However, the CLDs of the Bis-GMA/IBOMA and Bis-GMA/IBOMA/TEGDMA were lower than that of the Bis-GMA/TEGDMA. The IBOMA is considered more hydrophobic than TEGDMA, which would lead to lower values of WS; however, on the other hand, it presents low ability to form crosslink among the polymer chains. The IBOMA is a monomethacrylate, presents low polymerization degree and, therefore, has fewer sites for crosslink in the polymer chain in formation. ${ }^{27}$ 
In this study, the resins with IBOMA alone had lower $\mathrm{KH}$ when compared with the TEGDMA groups. This fact can be explained because monomethacrylates such as IBOMA tend to form linear polymers when polymerized alone or in resin blends, unlike what happens with the TEGDMA, which is known as conventional crosslinkers in polymers. ${ }^{9}$

Similar to the present study, Favarão et al said ${ }^{11}$ the IBOMA associated with TEGDMA showed good or intermediate physical and mechanical properties. Also, it could be an alternative to improve the organic matrix of the composites, since it showed similar KH values when compared with TEGDMA groups. However, it was promising mainly because it can reduce the polymerization contraction. The results of the present study corroborate other studies that show that experimental resin blends can be promising for the development new dental composites. ${ }^{12-16,27}$ However, further investigations should be conducted to clarify not only the durability of this type of resin blend, analyzing marginal adaptation and bond strength, but also get an interesting formulation for the dental practice. Another important factor is the inclusion of inorganic filler particles for evaluating the performance of IBOMA as reactive diluent monomer in dental resins.

\section{Conclusion}

The monomethacrylate with low-polymerization shrinkage IBOMA used alone or in combination with TEGDMA may decrease VS, WS and CLD values. Thus, it can be used as a blend for dental resins.

\section{Conflict of Interest}

None declared.

\section{References}

1 Kassardjian V, Andiappan M, Creugers NHJ, Bartlett D. A systematic review of interventions after restoring the occluding surfaces of anterior and posterior teeth that are affected by tooth wear with filled resin composites. J Dent 2020;99:103388 doi:10.1016/j.jdent.2020.103388

2 Gul P, Alp HH, Özcan M. Monomer release from bulkfill composite resins in different curing protocols. J Oral Sci 2020;62(3):288-292

3 Nagaoka H, Bishop S, Roberts H. Flexural performance of direct resin composite restorative materials past expiration date. Eur J Dent 2020;14(2):217-223

4 Barszczewska-Rybarek IM, Chrószcz MW, Chladek G. Novel urethane-dimethacrylate monomers and compositions for use as matrices in dental restorative materials. Int J Mol Sci 2020;21(7):2644

5 Palagummi SV, Hong T, Wang Z, Moon CK, Chiang MY. Resin viscosity determines the condition for a valid exposure reciprocity law in dental composites. Dent Mater 2020;36(2):310-319

6 Habib E, Wang R, Zhu XX. Correlation of resin viscosity and monomer conversion to filler particle size in dental composites. Dent Mater 2018;34(10):1501-1508

7 Fugolin AP, de Paula AB, Dobson A, et al. Alternative monomer for BisGMA-free resin composites formulations. Dent Mater 2020;36(7):884-892
8 Gonçalves F, Pfeifer CS, Ferracane JL, Braga RR. Contraction stress determinants in dimethacrylate composites. J Dent Res 2008;87(4):367-371

9 Altintas SH, Usumez A. Evaluation of TEGDMA leaching from four resin cements by HPLC. Eur J Dent 2012;6(3):255-262

10 Nie J, Lovell LG, Bowman CN. Synthesis and characterization of $\mathrm{N}$-isopropyl, N-methacryloxyethyl methacrylamide as a possible dental resin. Biomaterials 2001;22(6):535-540

11 Favarão J, Oliveira DCRS, Rocha MG, et al. Solvent degradation and polymerization shrinkage reduction of resin composites using isobornyl methacrylate. Braz Dent J 2019;30(3):272-278

12 Barot T, Rawtani D, Kulkarni P. Physicochemical and biological assessment of silver nanoparticles immobilized Halloysite nanotubes-based resin composite for dental applications. Heliyon 2020;6(3):e03601

13 Barot T, Rawtani D, Kulkarni P. Development of chlorhexidine loaded halloysite nanotube based experimental resin composite with enhanced physico-mechanical and biological properties for dental applications. J Compos Sci 2020;4:81

14 Barot T, Rawtani D, Kulkarni P, Hussain CM, Akkireddy S. Physicochemical and biological assessment of flowable resin composites incorporated with farnesol loaded halloysite nanotubes for dental applications. J Mech Behav Biomed Mater 2020;104:103675 doi:10.1016/j.jmbbm.2020.103675

15 Moraes RR, Garcia JW, Barros MD, et al. Control of polymerization shrinkage and stress in nanogel-modified monomer and composite materials. Dent Mater 2011;27(6):509-519

16 Liu J, Howard GD, Lewis SH, Barros MD, Stansbury JW. A study of shrinkage stress reduction and mechanical properties of nanogel modified resin systems. Eur Polym J 2012;48(11):1819-1828

17 He J, Liu F, Luo Y, et al. Properties of 2,2-Bis[p-(2'-hydroxy-3'methacryloxy propoxy)phenyl]propane/Isobornyl (Meth)acrylate copolymers. J Appl Polym Sci 2012;126:1527-1531

18 Sideridou ID, Karabela MM, Vouvoudi ECh. Dynamic thermomechanical properties and sorption characteristics of two commercial light cured dental resin composites. Dent Mater 2008;24(6):737-743

19 Pérez-Mondragón AA, Cuevas-Suárez CE, González-López JA, Trejo-Carbajal N, Meléndez-Rodríguez M, Herrera-González AM. Preparation and evaluation of a BisGMA-free dental composite resin based on a novel trimethacrylate monomer. Dent Mater 2020;36(4):542-550

20 std . ISO 4049:2000 Dentistry - polymer-based filling, restorative and luting materials; 7.10 Depth of cure, Class 2 materials. International Organization for Standardization; 2000/std

21 Braden M, Causton EE, Clarke RL. Diffusion of water in composite filling materials. J Dent Res 1976;55(5):730-732

22 Sideridou I, Tserki V, Papanastasiou G. Study of water sorption, solubility and modulus of elasticity of light-cured dimethacrylate-based dental resins. Biomaterials 2003;24(4):655-665

23 Cao W, Zhang Y, Wang X, et al. Novel resin-based dental material with anti-biofilm activity and improved mechanical property by incorporating hydrophilic cationic copolymer functionalized nanodiamond. J Mater Sci Mater Med 2018;29(11):162

24 Kemaloglu H, Pamir T, Tezel H. A 3-year randomized clinical trial evaluating two different bonded posterior restorations: Amalgam versus resin composite. Eur J Dent 2016;10(1):16-22

25 Prakki A, Tallury P, Mondelli RF, Kalachandra S. Influence of additives on the properties of Bis-GMA/Bis-GMA ana$\log$ comonomers and corresponding copolymers. Dent Mater 2007;23(10):1199-1204

26 Asmussen E, Peutzfeldt A. Influence of selected components on crosslink density in polymer structures. Eur J Oral Sci 2001b;109(4):282-285

27 Cui Y, Yang J, Zhaohua Z, et al. Unique morphology and properties study of polyacrylate obtained via frontal photopolymerization. Polymer 48(20):5994-6001 